\title{
The ionospheric perturbations associated with Asian earthquakes as seen from the subionospheric propagation from NWC to Japanese stations
}

\author{
Y. Kasahara ${ }^{1,2}$, F. Muto ${ }^{1,2}$, Y. Hobara ${ }^{1,2}$, and M. Hayakawa ${ }^{2,3}$ \\ ${ }^{1}$ University of Electro-Communications(UEC), Department of Electronic Engineering, 1-5-1 Chofugaoka, \\ Chofu Tokyo, 182-8585, Japan \\ ${ }^{2}$ UEC, Research Station on Seismo Electromagnetics, Chofu Tokyo, Japan \\ ${ }^{3}$ UEC, Advanced Wireless Communications Research Center, Chofu Tokyo, Japan
}

Received: 11 December 2009 - Revised: 11 March 2010 - Accepted: 12 March 2010 - Published: 26 March 2010

\begin{abstract}
Huge five earthquakes with magnitude greater than 6.0 took place in Asia (include Philippines, Indonesia, etc.) during the period from the beginning of August 2008 to the end of Junuary 2009, and the corresponding data of subionospheric VLF propagation between the NWC transmitter (Australia, $19.8 \mathrm{kHz}$ ) and a few Japanese stations (distance $6 \sim 8 \mathrm{Mm}$ ) are examined. As the result of our analysis by means of (1) trend (average nighttime amplitude), (2) dispersion, (3) nighttime fluctuation, and (4) atmospheric gravity wave enhancement, three earthquakes from the five taking place within the fifth Frenel zone are found to have accompanied a precursory signature in VLF propagation. On the other hand, there were observed no such precursory signatures for the remaing two earthquakes. One is too deep $(>400 \mathrm{~km})$ and another is too distant from the great-circle path. These characteristics of seismo-ionospheric perturbations would be of essential importance in studying the spatial/temporal properties of seismo-ionospheric perturbations for medium-distance propagation.
\end{abstract}

\section{Introduction}

Based on the extensive studies on electromagnetic effects associated with earthquakes (EQs) during the last decade (Hayakawa, 1999, 2009; Hayakawa and Molchanov, 2002; Molchanov and Hayakawa, 2008), it is recently agreed that the ionosphere is definitely perturbed prior to an EQ both in its lower part (D/E region) (e.g., Hayakawa, 2007) and also in its upper region (F region) (e.g., Liu et al., 2006).

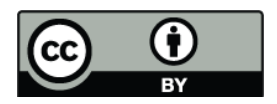

Correspondence to: Y. Kasahara (hayakawa@whistler.ee.uec.ac.jp)
We have been working on collecting a lot of events on ionospheric perturbations in possible association with EQs as based on the use of subionospheric VLF/LF transmitter signals (e.g., Hayakawa, 2007). There have been published case studies for huge EQs including the 2003 Tokachi-oki EQ (Shvets et al., 2004; Hayakawa et al., 2005), the 2004 Niigata-chuetsu EQ (Hayakawa et al., 2006), the Sumatra EQ (Horie et al., 2007a, b), the 2008 Miyagi-oki EQ (Muto et al., 2008,2009; Rozhnoi et al., 2007), etc. Also very few statistical studies have been reported on the definitely significant correlation betweenVLF/LF propagation anomalies and EQs with magnitude greater than 6.0 and shallow depth (Rozhnoi et al., 2004; Maekawa et al., 2006; Kasahara et al., 2008).

A majority of above papers are based on the data over relatively short distance (distance is from $1 \mathrm{Mm}$ to a few $\mathrm{Mm}$ ) propagation. We have presented a very convincing evidence on ionospheric perturbations associated with the Kobe EQ (Hayakawa et al., 1996), and this finding was based on the daily trace of the terminator times (TTs) by using the Omega VLF transmitter located at Tsushima and by receiving this signal at Inubo. The TT is defined as a time when the diurnal variation of amplitude (or phase) shows a minimum around the local sunset or sunrise. The distance between the VLF transmitter and receiver for the Kobe case is only $1 \mathrm{Mm}$, and we have interpreted the shift in TTs in term of the lowering of the lower ionosphere before the EQ (Hayakawa et al., 1996; Molchanov and Hayakawa et al., 1998; Yoshida et al., 2008). The shift of this TT effect has been investigated by Clilverd et al. (1999) for an extremely long (more than $10 \mathrm{Mm}$ ) NS propagation path, and concluded that there existed no seismoionospheric perturbation and that the TT method is not useful in finding out seismo-ionospheric perturbations. It is rather easy for us to consider that it is reasonable for them to have detected no TT anomaly on their long propagation path on

Published by Copernicus Publications on behalf of the European Geosciences Union. 
Table 1. EQs treated in this paper.

\begin{tabular}{|c|c|c|c|}
\hline $\begin{array}{l}\text { Date } \\
\text { (UT) }\end{array}$ & $\begin{array}{l}\text { Geographic } \\
\text { coordinates }\end{array}$ & Magnitude & $\begin{array}{l}\text { Depth } \\
{[\mathrm{km}]}\end{array}$ \\
\hline $\begin{array}{l}15 \text { Aug } 2008 \\
(10: 25: 16)\end{array}$ & $\begin{array}{l}12.90^{\circ} \mathrm{N} 124.32^{\circ} \mathrm{E} \\
\text { Philippines }\end{array}$ & 6 & 10 \\
\hline $\begin{array}{l}11 \text { Sep } 2008 \\
(00: 00: 02)\end{array}$ & $\begin{array}{l}1.88^{\circ} \mathrm{N} 127.36^{\circ} \mathrm{E} \\
\text { Indonesia }\end{array}$ & 6.6 & 96 \\
\hline $\begin{array}{l}16 \text { Nov } 2008 \\
(17: 02: 32)\end{array}$ & $\begin{array}{l}1.27^{\circ} \mathrm{N} 122.09^{\circ} \mathrm{E} \\
\text { Indonesia }\end{array}$ & 7.3 & 30 \\
\hline $\begin{array}{l}\text { 6 Dec } 2008 \\
10: 55: 26\end{array}$ & $\begin{array}{l}7.39^{\circ} \mathrm{S} 124.73^{\circ} \mathrm{E} \\
\text { East Timor }\end{array}$ & 6.4 & 404 \\
\hline $\begin{array}{l}3 \text { Jan } 2009 \\
22: 33: 40\end{array}$ & $\begin{array}{l}0.41^{\circ} \mathrm{S} 132.89^{\circ} \mathrm{E} \\
\text { Papua New Guinea }\end{array}$ & 7.6 & 17 \\
\hline
\end{tabular}

the following reasons. The first reason is that the perturbed region is too small as compared with the whole propagation path to expect any propagation anomaly. The second reason is that their NS propagation itself is not so suitable generally to detect any TT effect (Maekawa and Hayakawa, 2007).

In this paper we pay attention to a medium distance (about $6 \sim 8 \mathrm{Mm}$ ) propagation path and we focus on several EQs for this distance propagation path. Unlike the above-mentioned TT method to identify seismo-ionospheric effect, we use an alternative method (we call it "nighttime fluctuation" method) (Rozhnoi et al., 2004; Maekawa et al., 2006; Horie et al., 2007a) for the VLF analysis in this paper, in order to examine whether we will be able to detect any seismoionospheric perturbation for the medium-distance propagation and to study the dependence of seismo-ionospheric disturbances on the distance of an EQ epicenter from the greatcircle path, the effect of EQ depth, etc.

\section{Analysis propagation paths and EQs treated}

In this paper we focus on the propagation paths associated with the Australian VLF transmitter (with call sign of NWC) located at North-west cape of Australia and with its transmitter frequency of $19.8 \mathrm{kHz}$. Three observing (or receiving) stations from the Japanese VLF/LF network (Hayakawa, 2007) have been used; Moshiri (abbreviated as MSR), Kochi $(\mathrm{KCH})$, and Chofu (CHF) as shown in Fig. 1. The distance from the transmitter (NWC) is $6.5 \mathrm{Mm}$ for $\mathrm{KCH}, 6.9 \mathrm{Mm}$ for CHF and $7.8 \mathrm{Mm}$ for MSR, respectively.

The period of data analysis is approximately six months from 1 August 2008 to 10 January 2009.

During this time period we had relatively a large number of EQs in the Asian countries. Five large EQs took place, and we list them in Table 1 (including the date (and time), epicentral position (and country), EQ magnitude and depth). The positions of epicenters of those EQs are plotted in Fig. 1,

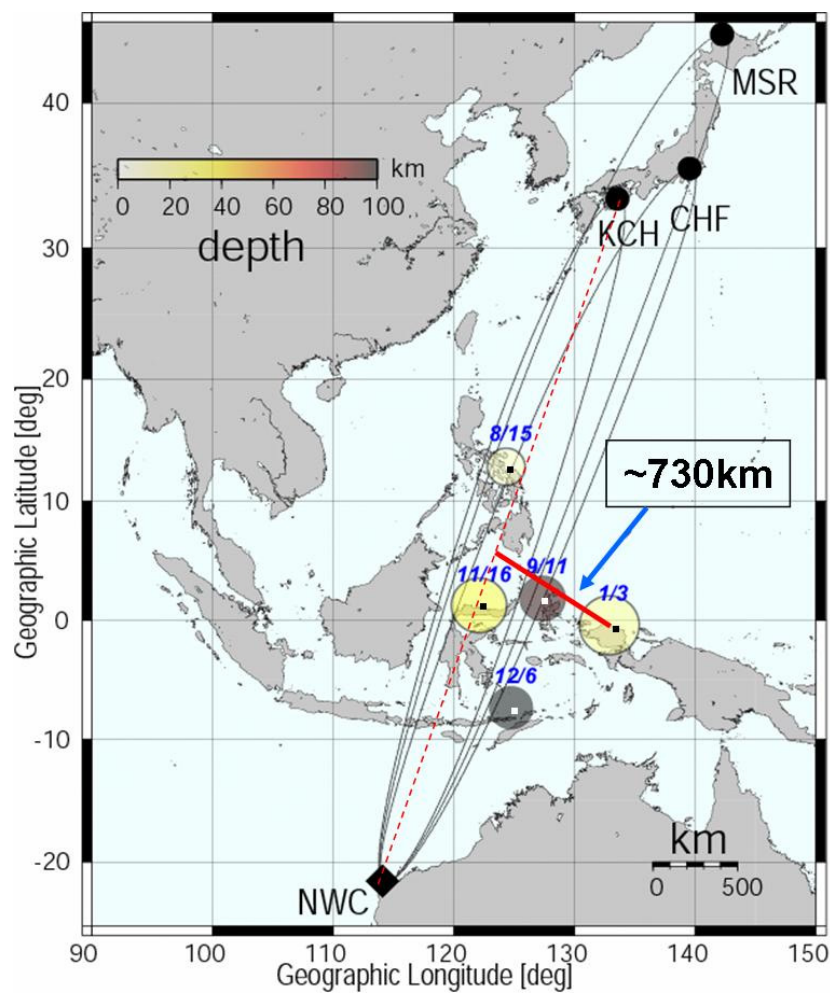

Fig. 1. The relative locations of the transmitter, NWC and some Japanese receiving stations (Moshiri (MSR), and Chofu (CHF) and Kochi $(\mathrm{KCH})$ ), together with the relevant wave sensitive area (defined by fifth Fresnel zone). Also, the epicenters of five EQs are indicated, together with the information on the EQ depth (in color)

together with the information on EQ depth (in color). The corresponding wave sensitive area in terms of the fifth Fresnel zone is also plotted in Fig 1 for each propagation path.

\section{VLF data analysis}

Our conventional analysis method based on the concept of nighttime fluctuation by using the local nighttime data is adopted (Rozhnoi et al., 2004; Maekawa et al., 2006; Kasahara et al., 2007; Muto et al., 2009), so that we analyze (1) trend (as the average nighttime amplitude), (2) dispersion, (3) N.F. (nighttime fluctuation) as the degree of fluctuation and (4) AGW (atmospheric gravity wave) modulation index. The first three physical and statistical quantities have been used extensively in our previous papers by Maekawa et al. (2006), Kasahara et al. (2008), Muto et al. (2008) in order to find out the presence of seismo-ionospheric perturbations, but the last parameter of AGW modulation index is recently suggested to be analyzed (Muto et al., 2009) in order to confirm the presence of seismo- ionospheric perturbations which seems to be closely related to their generation mechanism (or lithosphere - atmosphere - ionosphere coupling). 


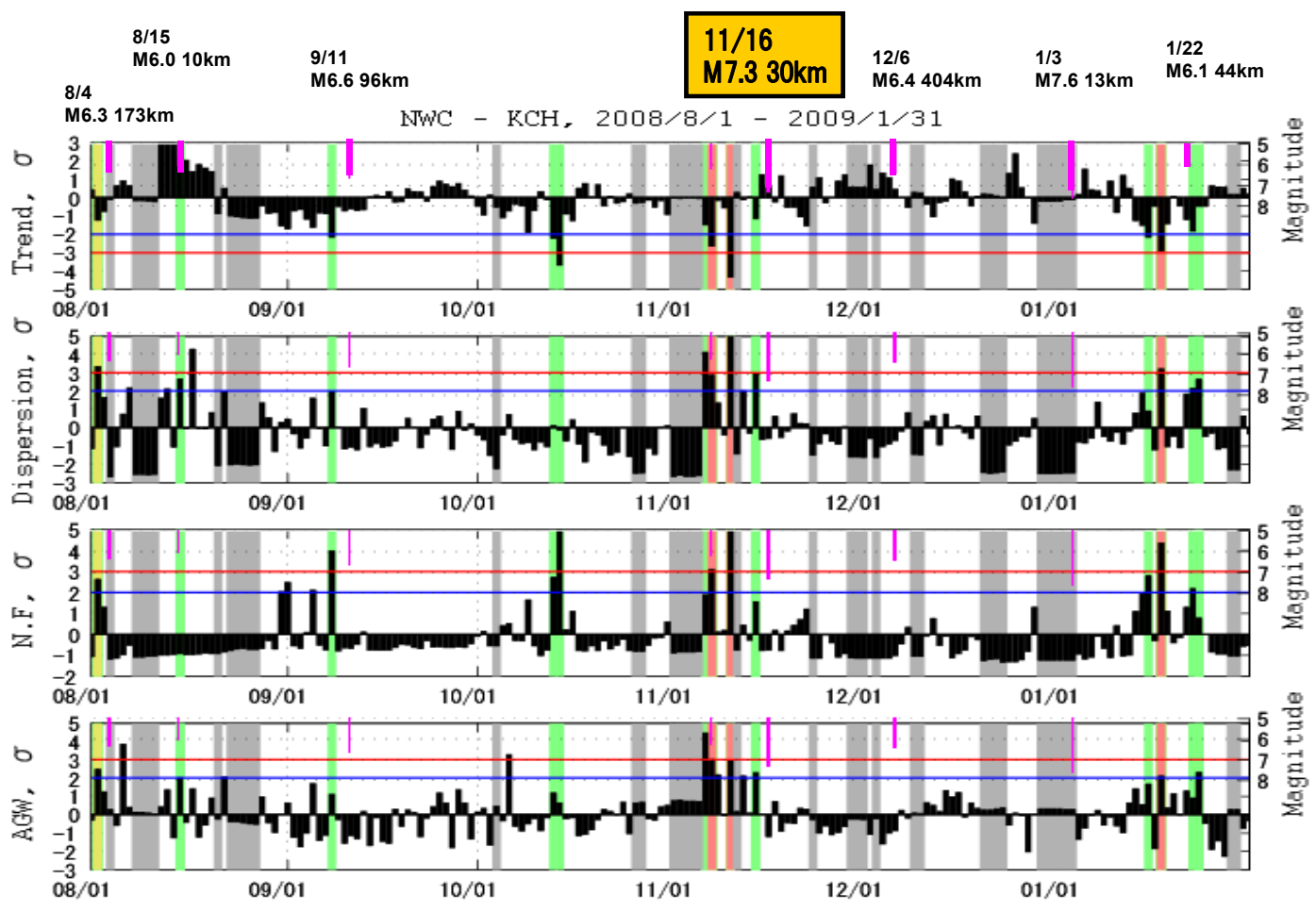

Fig. 2. Temporal evolution of the 3 physical parameters (top, trend, second, dispersion, third, N.F.) for the path from NWC to KCH and an additional parameter of AGW at the bottom. All quantities are normalized with respect to their corresponding standard deviation and the important thresholds are indicated by a horizontal blue line $(2 \sigma)$ and a horizontal red line $(3 \sigma)$. The dates of EQs are indicated on the top in yellow. A red box means that all of the 4 physical parameters satisfy their $2 \sigma$ criteria, while a yellow box means that 3 physical parameters satisfy their $2 \sigma$ criteria and a green box means that 2 physical parameters satisfy their $2 \sigma$ criteria. Grey areas indicate the lack of observation, so that there are no data.

The details on the definition of these parameters have already been described in our former papers (Maekawa et al., 2006; Kasahara et al., 2008; Muto et al., 2008, 2009), but some essential points are repeated here. The diurnal variation of the signal amplitude is described by $A(t)$, which is the amplitude at a time $t$ on a particular day. Then $\langle A(t)>$ is defined as the average value at the same time $t$ over 1 to -30 days of the current day. We take a difference $d A(t)=A(t)-<A(t)>$, which tends to reduce the longterm (e.g. seasonal) variation and which is related with the short-term effect. Unlike the TT method, the present nighttime fluctuation method is based on the analysis only during local nighttime (e.g., UT=13:00-18:00 h for NWC-KCH, $\mathrm{UT}=12: 00-17: 00 \mathrm{~h}$, both for NWC-CHF and NWC-MSR). The first parameter, trend is the mean value of $d A(t)$ for each day. Second parameter, dispersion is a purely statistical quantity of dispersion of $d A(t)$ for each day. The third parameter, N.F. is defined by the area of $d A(t)<0$ integrated over the whole nighttime. The final AGW modulation index is estimated in the following way (Muto et al., 2009). The $d A(t)$ for each day (one night) is subjected to FFT, and we estimate $d S(f)=S(f)-<S(f)>$ for each day in which $S(f)$ is the FFT spectrum on the current day and $\langle S(f)>$ is the means spectrum over 1 to 30 days before the relevant day. This fluctuation spectrum in the range of period from $10 \mathrm{~min}$ to $100 \mathrm{~min}$ (corresponding to atmospheric gravity waves, AGWs) is our interest and the area of $d S(f)>0$ over the relevant period interval is the AGW modulation index. These four parameters are normalized with respect to their corresponding standard deviations. Here we emphasize that the most important parameter is the trend, which shows a significant depletion before an EQ, and the 2nd (dispersion) and 3rd (N.F.) parameters are of the secondary important ones, which show increases before the EQ, as is already shown in Maekawa et al. (2006) and Kasahara et al. (2008).

\section{Analysis results}

Analysis results are presented in Figs. 2, 3, and 4. Figure 2 refers to the path from NWC to $\mathrm{KCH}$, Fig. 3 from $\mathrm{NWC}$ to CHF, Fig. 4 from NWC to MSR, respectively.

By taking Fig. 2 as an example, we need to explain what are described in the figure. You notice four panels in the figure: from the top to the bottom, trend, dispersion, N.F. and AGW modulation index. The ordinate for each parameter is given in terms of its standard deviation $(\sigma)$, so that we 


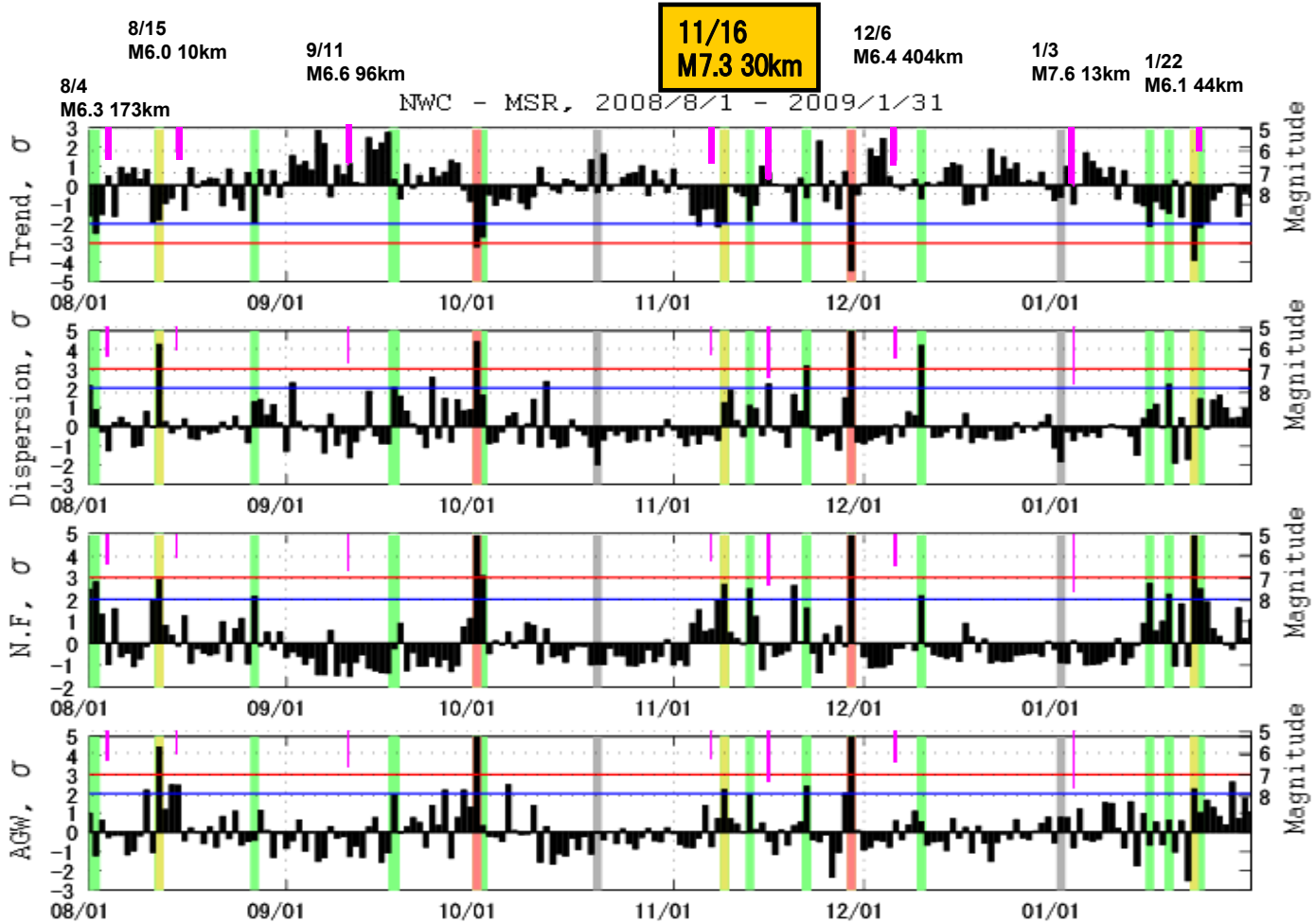

Fig. 3. The same as Fig. 2, but for the path of NWC-MSR.

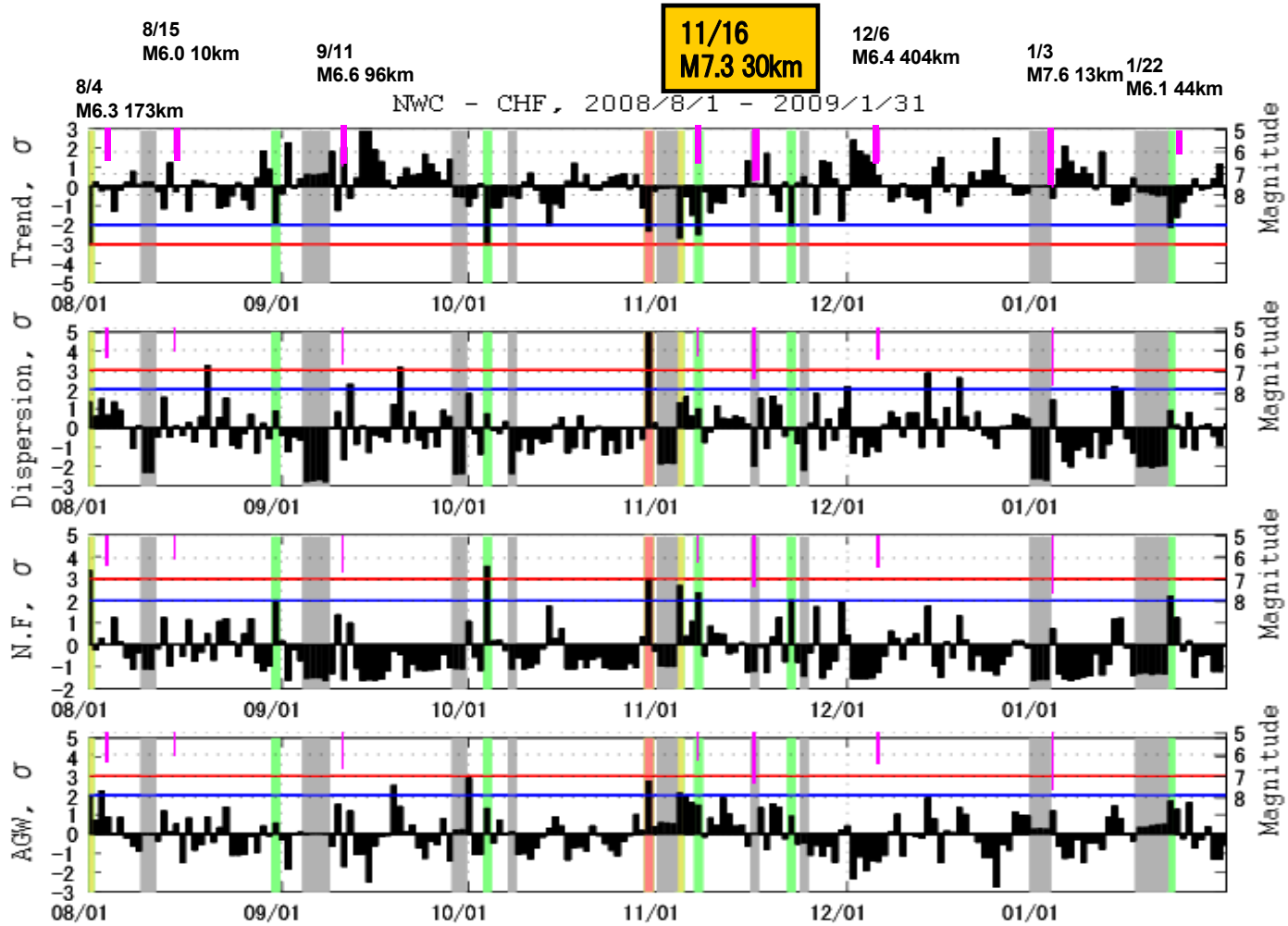

Fig. 4. The same as Fig. 2, but for the path of NWC-CHF. 
normally pay attention to $\pm 2 \sigma$ as a criterion. The important threshold for trend is given by a horizontal blue line $(-2 \sigma)$ and a horizontal red line $(-3 \sigma)$. Similarly the corresponding thresholds for dispersion, N.F. and AGW are given by the same color horizontal lines. The date is given on the bottom of each panel, starting from 1 August 2008 to the end of January 2009. Five EQs in Table 1 are described in the top of the first panel (including the date, EQ magnitude and depth), and the occurrence times of those EQs are indicated by downward red bars in the top of each panel (its length is proportional to EQ magnitude according to the right scale of each panel). The main EQ with the biggest $\mathrm{EQ}$ is given in a yellow box as well. In addition to these $5 \mathrm{EQs}$, there are also indicated two significant EQs in the beginning and in the end of our analysis period; 4 August 2008 and 22 January 2009 EQs. The period in gray indicates the period of no observation due to different problems, so that there are no data there. As is already known from Rozhnoi et al. (2004), Maekawa et al. (2006), Kasahara et al. (2008), and Muto et al. $(2008,2009)$, the trend tends to decrease down to $-2 \sigma$ level, while three other parameters tend to be enhanced over their corresponding $2 \sigma$ criteria. When we satisfy these criteria, the period is likely to be seismogenic and it would be a precursory signature of an EQ. In the figure there are three different colors of boxes; A red box (or day, or days) means that all of the four parameters satisfy the $2 \sigma$ criteria for that day, a yellow box means that three of the four parameters satisfy their $2 \sigma$ level, and finally a green box indicates that two of the four parameters satisfy their $2 \sigma$ level. So we are ready to look at each carefully and we try to understand whether there existed any precursory EQ signature or not.

We look at Fig. 2 for the path from NWC-KCH. Though there are a lot of periods with data gaps (in gray), we will try to find out any propagation anomalies. Though there are some data gaps before the 1st EQ on 15 August, we could find out an anomaly about 10 days before the EQ (indicated by a yellow box), which would appear to be a precursor to the EQ. You see a green box on the EQ day, but the 1st parameter, trend did not decrease, which means that this is not seismogenic. Then, before the 2nd EQ on 11 September, there is present a clear precursor a few days before the $\mathrm{EQ}$ (both a significant decrease in trend and an increase in dispersion (approaching $+2 \sigma$ level) and N.F.). As for the 3rd EQ, the biggest EQ in this area on 16 November, we could find very conspicuous propagation anomalies (in two red boxes) on two days as a series about one week before the EQ, which are highly likely to be precursors of this EQ. You can notice a green box about one month before the EQ on 16 November, for which we have observed a significant decrease in the trend, but not so much in dispersion. This additional anomaly is not found to accompany an EQ. We will come to this anomaly later after looking at the same anomaly for other two propagation paths. As for the next EQ on 6 December, we could not find out any conspicuous propagation anomaly. This is reasonably acceptable because of a large EQ depth of $404 \mathrm{~km}$, as based on our previous works (Molchanov and Hayakawa, 1998; Maekawa et al., 2006). Finally, we try to find any anomaly before the last EQ on 3 January, which is strong enough $(M=7.6)$ and shallow enough. However there is no propagation anomaly, which is due to the fact that the EQ epicenter is far away $(>700 \mathrm{~km})$ from the propagation path.

Next we move on to the next result in Fig. 3 for the propagation path from NWC to MSR. Concerning the first EQ on 15 August, there were observed two precursory VLF propagation anomalies a few days (yellow box) and about two weeks (green box) before the EQ. The anomaly in a yellow box is highly likely to be a precursor, but it is not definite whether the anomaly in a green box is related to the EQ on 15 August or another earlier EQ on 4 August. We move on to the next EQ on 11 September. About two weeks before the $\mathrm{EQ}$, we can notice the presence of a green box, in which the trend is decreased exceeding $-2 \sigma$ level and the dispersion is enhanced (but not exceeding $2 \sigma$ level). This is considered to be a very clear anomaly, which might be reasonably acceptable because of a deep EQ with depth of $\sim 100 \mathrm{~km}$.

Then, there is a very clear VLF propagation anomaly on 1 October, expressed in a red and a green box. There is no definite EQ just around this propagation anomaly, so that we will consider it later again. Next we discuss our main event of 16 November EQ with magnitude of 7.3 and with a shallow depth of $30 \mathrm{~km}$. The following EQ occurred about 20 days later on 5 December. However, the depth of the later EQ is $404 \mathrm{~km}$, and this means that no ionospheric anomaly is expected for this EQ on the basis of our previous experience. So that, it is very reasonable for us to suppose that a series of VLF propagation anomalies from 10 November to just before the end of December is due to the main EQ on 16 November (that is, a precursor effect about one week before the EQ (expressed by a yellow box) and its after-effects). Finally, as for the EQ on 3 January, there is no definite precursor to this EQ.

Final figure, Fig. 4 for the path of NWC-CHF is discussed now. As for the first EQ on 15 August, there was observed a clear propagation anomaly (in a yellow box) about two weeks before the $\mathrm{EQ}$, which is not a precursor to the earlier EQ on 4 August, but is a precursor to the EQ on 15 August. This anomaly is common to other propagation paths. We move on to the 2nd EQ on 11 September. We can notice an anomaly in green box about 10 days before the EQ, but the trend showed a significant decrease (exceeding $-2 \sigma$ level), but the dispersion is only slightly enhanced (not exceeding $2 \sigma$ level). This one is, hence, not considered to be a clear anomaly, and this is closely related with the deep depth of this EQ. Again, we notice an anomaly in green box in the early October, without any EQ, which will be discussed later again. Then, we move on to the major EQ on 16 November. It seems that the period of two weeks before the EQ to about one week after the EQ, is a series of effects associated with this EQ. That is, starting from the beginning of November and lasting for one week, 
Table 2. Summary on the propagation anomaly for the relevant five EQs. A circle means the presence of an anomaly, and a cross, the obsence of the anomaly. A bar means that some period of no observation might have an influence so that the result is not so certain. A question means that an anomaly (it did exist) is likely to be the after-effect of the previous EQ.

\begin{tabular}{cccccc}
\hline & 15 Aug 2008 & 11 Sep 2008 & 16 Nov 2008 & 6 Dec 2008 & 3 Jan 2009 \\
& $M 6.010 \mathrm{~km}$ & $M 6.696 \mathrm{~km}$ & $M 7.330 \mathrm{~km}$ & $M 6.4404 \mathrm{~km}$ & $M 7.617 \mathrm{~km}$ \\
\hline NWC-CHF & $\bigcirc(-)$ & $\bigcirc$ & $\bigcirc(-)$ & - & $\mathrm{X}$ \\
NWC-MSR & $\bigcirc$ & $\bigcirc$ & $\bigcirc$ & $\bigcirc(?)$ & $\mathrm{X}$ \\
NWC-KCH & $\bigcirc(-)$ & $\bigcirc(-)$ & $\bigcirc(-)$ & $\bigcirc(?)$ & $\mathrm{X}$ \\
\hline
\end{tabular}

we could observe a series of propagation anomalies (one red, one yellow and one green), which is definitely a precursor to this EQ. Afterwards, there was a definite after-effect, about one week after the EQ. This kind of simultaneous occurrence of not only a precursor, but also after-effect is very peculiar to a huge EQ like this EQ (see Shvets et al. (2004) for the 2003 Tokachi-oki EQ, Hayakawa et al. (2006) for the 2004 Niigata-chuetsu EQ, etc.). The temporal separation of the major EQ and the following EQ on 6 December is only 20 days, so that it is rather difficult to distinguish between the after-effect of the previous EQ and a precursor to the following EQ. However, our previous paper says that no propagation anomaly is expected for a deep EQ like this. Finally, it seems that there is no precursor to the last EQ on 3 January.

\section{Conclusion and discussion}

Based on the observation of VLF signals from the NWC transmitter in Australia at a few Japanese stations for several EQs in the Asian region, we have come to the following conclusions. We will summarize the observational results for each EQ one by one, which is given in Table 2. In the table a circle means the presence of any anomaly, and a cross indicates the absence of the anomaly. A bar means some uncertainty due to the lack of observation. A question mark indicates a possibility of the after - effect of the previous EQ.

\section{August EQ in Philippines}

Even though the data quality was not so good at the three station (KCH, CHF, and MSR) because of a lot of no measurement periods, it seems clear that a definite precursor is observed about two weeks before the EQ and no after-effect is detected at all three stations. Based on our previous experience, the depth of $\sim 100 \mathrm{~km}$ is too large to expect an anomaly, but the EQ magnitude is big, 6.6. So that, the parameters of this EQ seem to lie at the boundary of having a VLF/LF propagation anomaly.

\section{September EQ}

A few days before the EQ, there was observed an anomaly at $\mathrm{KCH}$, but no clear precursor is seen at MSR. There is a long data gap at CHF before the EQ, so that it is very uncertain whether there is an anomaly at CHF or not.

\section{Major EQ on 16 November}

There exist very clear and definite precursory propagation anomalies at all of the three stations one to two weeks before the EQ. The duration is considerably long, of the order of one week. Together with this precursory signature, there is observed an after-effect as well.

\section{December EQ}

There existed some anomalies, but our previous study suggests that such a deep EQ induces no precursory anomaly, so that the anomaly observed might be attributed to the previous big EQ as its after - effect.

\section{January EQ}

It is definite that any precursory effect is not seen at all the three stations, because of the far distance of EQ epicenter from three propagation paths, even though the magnitude is too big and the depth is too shallow.

The purpose of this paper is to demonstrate that even the medium-distance $(6 \sim 8 \mathrm{Mm})$ propagation can be used to identify the propagation anomaly (or ionospheric perturbation) in possible association with EQs, which is considered to be a response to the question raised by Clilverd et al. (1999). We have developed two possible ways of analysis for subionosphric VLF/LF propagation; (1) TT method (initially proposed by Hayakawa et al., 1996) and (2) nighttime fluctuation method (Rozhnoi et al., 2004; Maekawa et al., 2006; Hayakawa, 2007).

Finally, we here comment again on a likely anomaly in the beginning of October. A red box is observed in MSR, which indicates a significant decrease in trend and significant increases in dispersion and N.F. This is very seismogenic. The anomaly is not so clear in CHF, with a significant decrease in 
trend. Again, the $\mathrm{KCH}$ data exhibited a significant increase in dispersion. Also, the occurrence date is slightly delayed with respect to the ones in MSR and CHF. Recently Muto et al. (2009) have found that there may exist the effect of Earth's tides one month (and two months) only before a huge EQ. Hayakawa et al. (2009) have done further extensive studies on the general seismo-electromagnetic effects and have confirmed the presence of such effects of Earth's tide. In this sense, the likely anomaly in the beginning of October might be a plausible evidence of Earth's tide.

As is already studied by Maekawa and Hayakawa (2007), TT method is found to be not so useful for the NS (or meridional) propagation direction as is easily anticipated. So that, the next method (nighttime fluctuation) seems to be a promising way to find out seismo-ionospheric perturbations. The present paper shows that this nighttime fluctuation method is of great potential to identify the ionospheric perturbations associated with EQs. The propagation distance is of the order of $6 \sim 8 \mathrm{Mm}$, probably the medium-distance in VLF, with respect to the long distance of $>10 \mathrm{Mm}$ in Clilverd et al. (1999) and the short distance ( $\sim 1 \mathrm{Mm})$ in Hayakawa et al. (1996).

Then, the characteristics of nighttime fluctuation are summarized for several EQs occurred in the Asian region. The nighttime fluctuation method is based on the measurement of three physical parameters ((i) trend, nighttime average amplitude; (ii) dispersion; (ii) nighttime fluctuation, N.F.; and (iv) AGW modulation).

1. The nighttime fluctuation method is found to be useful to identify seismo-ionospheric perturbations on the basis of medium-distance $(6 \sim 8 \mathrm{Mm})$ subionospheric propagation data even in the NS propagation (for which TT method is not so effective). An anomaly is characterized by a decrease in trend and enhancements in dispersion and N.F., just like for short-distance properties by Maekawa et al. (2006) and Kasahara et al. (2008).

2. When the EQ epicenter is located within the wave sensitive area (or fifth Fresnel zone) of the propagation path, we can definitely detect a propagation anomaly when the EQ magnitude is greater than 6.0 and its depth is shallow (10 km in this paper). The anomaly appears only as a precursor, without any after-effect.

3. When there occurs a huge EQ with magnitude of 6.6, but its depth is rather large, $96 \mathrm{~km}$, there appears a propagation anomaly on one path, but no anomaly is seen on other propagation paths. This combination of EQ parameters (magnitude and depth) seems to be the boundary of having the VLF anomaly.

4. The main EQ on 16 November, showed the following propagation characteristics. This EQ is extremely large with magnitude of 7.3 and also very shallow $(30 \mathrm{~km})$. The resulting anomaly is characterized by a prolonged ( one week) precursory signature about one to two weeks before the EQ. Together with this, it seems that there is an after-effect. Further, the effect of Earth's tides seems to appear one month before this EQ.

5. A huge (M6.4), but a deep (404 km) EQ seems to have induced no propagation anomaly.

6. The 3 January EQ had a large magnitude of 7.6 and shallow (depth $13 \mathrm{~km}$ ), which showed no definite anomaly. This is probably due to the reason that the EQ epicenter is far away from the propagation path $(700 \sim 800 \mathrm{~km})$ and it is not located within its wave sensitive area.

These characteristics of possible seismo-ionospheric perturbations would be of essential importance in studying further details of the spatial/temporal properties of seismo-ionospheric perturbations and then in studying their generation mechanism in term of the concept of lithosphere-atmosphere-ionosphere coupling (Molchanov and Hayakawa, 2008; Hayakawa, 2009).

Acknowledgements. The authors would like to thank National Institute of Information and Communications Technology (NICT) for its support.

Edited by: M. E. Contadakis

Reviewed by: two anonymous referees

\section{References}

Clilverd, M. A., Rodger, C. J., and Thomson, N. R.: Investigating seismo-ionospheric effects on a long subionospheric path, J. Geophys. Res., 104(A12), 28171-28179, 1999.

Hayakawa, M. (Ed.): Atmospheric and Ionospheric Electromagnetic Phenomena Associated with Earthquakes, Terra Sci. Pub. Co., Tokyo, 996 pp., 1999.

Hayakawa, M.: VLF/LF radio sounding of ionospheric perturbations associated with earthquakes, Sensors, 7, 1141-1158, 2007.

Hayakawa, M. (Ed.): Electromagnetic Phenomena Associated with Earthquakes, Transworld Research Network, Trivandrum (India), 279 pp., 2009.

Hayakawa, M. and Molchanov, O. A. (Eds.): Seismo Electromagnetics: Lithosphere - Atmosphere - Ionosphere Coupling, TERRAPUB, Tokyo, 477 pp., 2002.

Hayakawa, M., Molchanov, O. A., Ondoh, T., and Kawai, E.: The precursory signature effect of the Kobe earthquake on VLF subionospheric signals, J. Comm. Res. Lab., Tokyo, 43, 169180, 1996.

Hayakawa, M., Ohta, K., Maekawa, S., Yamauchi, T., Ida, Y., Gotoh, T., Yonaiguchi, N., Sasaki, H., and Nakamura, T.: Electromagnetic precursors to the 2004 Mid Niigata Prefecture earthquake, Phys. Chem. Earth, 31, 356-364, 2006.

Hayakawa, M., Shvets, A. V., and Maekawa, S.: Subionospheric LF monitoring of ionospheric perturbations prior to the Tokachi-oki earthquake and a possible mechanism to lithosphere - ionosphere coupling, Adv. Polar. Up. Atmos. Res., 19, 42-54, 2005. 
Hayakawa, M., Sue, Y., and Nakamura, T.: The effect of earth tides as observed in seismo-electromagnetic precursory signals, Nat. Hazards Earth Syst. Sci., 9, 1733-1741, 2009, http://www.nat-hazards-earth-syst-sci.net/9/1733/2009/.

Horie, T., Maekawa, S., Yamauchi, T., and Hayakawa, M.: A possible effect of ionospheric perturbations associated with the Sumatra earthquake, as revealed from subionospheric very-lowfrequency (VLF) propagation (NWC-Japan), Int. J. Remote Sens., 28(13), 3133-3139, 2007a.

Horie, T., Yamauchi, T., Yoshida, M., and Hayakawa, M.: The wave-like structures of ionospheric perturbation associated with Sumatra earthquake of 26 December 2004, as revealed from VLF observation in Japan of NWC signals, J. Atmos. Sol.-Terr. Phy., 69, 1021-1028, 2007b.

Kasahara, Y., Muto, F., Horie, T., Yoshida, M., Hayakawa, M., Ohta, K., Rozhnoi, A., Solovieva, M., and Molchanov, O. A.: On the statistical correlation between the ionospheric perturbations as detected by subionospheric VLF/LF propagation anomalies and earthquakes, Nat. Hazards Earth Syst. Sci., 8, 653-656, 2008, http://www.nat-hazards-earth-syst-sci.net/8/653/2008/.

Liu, J. Y., Chen, Y. I., and Chuo, Y. J.: A statistical investigation of pre earthquake ionospheric anomaly, J. Geophys. Res., 111, A05304, doi:10.1029/2005JA011333, 2006.

Maekawa, S. and Hayakawa, M.: A statistical study on the dependence of characteristics of VLF/LF terminator, IEEJ T. Fund. Mater., 126(4), 220-226, 2006.

Maekawa, S., Horie, T., Yamauchi, T., Sawaya, T., Ishikawa, M., Hayakawa, M., and Sasaki, H.: A statistical study on the effect of earthquakes on the ionosphere, based on the subionospheric LF propagation data in Japan, Ann. Geophys., 24, 2219-2225, 2006, http://www.ann-geophys.net/24/2219/2006/.

Molchanov, O. A. and Hayakawa, M.: Seismo Electromagnetics and Related Phenomena: History and latest results, TERRAPUB, Tokyo, 189 pp., 2008.

Molchanov, O. A. and Hayakawa, M.: Subionospheric VLF signal perturbations possibly related to earthquakes, J. Geophys. Res., 103, 17489-17504, 1998.

Molchanov, O. A. and Hayakawa, M.: Seismo Electromagnetics and Related Phenomena: History and latest results, TERRAPUB, Tokyo, 189 pp., 2008.
Muto, F., Yoshida, M., Horie, T., Hayakawa, M., Parrot, M., and Molchanov, O. A.: Detection of ionospheric perturbations associated with Japanese earthquakes on the basis of reception of LF transmitter signals on the satellite DEMETER, Nat. Hazards Earth Syst. Sci., 8, 135-141, 2008, http://www.nat-hazards-earth-syst-sci.net/8/135/2008/.

Muto, F., Horie, T., Yoshida, M., Hayakawa, M., Rozhnoi, A., Solovieva, M., and Molchanov, O. A.: Ionospheric perturbations related to the Miyagi-oki earthquake on 16 August 2005, as seen from Japanese VLF/LF subionospheric propagation network, in: Special issue: Electromagnetic Phenomena Associated with Earthquakes and Volcanoes, edited by: Hayakawa, M., Liu,J. Y., Hattori, K., and Telesca, L., Phys. Chem. Earth, Pts. A/B/C, 34(6-7), 449-455, 2009.

Rozhnoi, A., Solovieva, M. S., Molchanov, O. A., and Hayakawa, M.: Middle latitude $\mathrm{LF}(40 \mathrm{kHz})$ phase variations associated with earthquakes for quiet and disturbed geomagnetic conditions, Phys. Chem. Earth, 29, 589-598, 2004.

Rozhnoi, A., Molchanov, O., Solovieva, M., Gladyshev, V., Akentieva, O., Berthelier, J. J., Parrot, M., Lefeuvre, F., Hayakawa, M., Castellana, L., and Biagi, P. F.: Possible seismo-ionosphere perturbations revealed by VLF signals collected on ground and on a satellite, Nat. Hazards Earth Syst. Sci., 7, 617-624, 2007, http://www.nat-hazards-earth-syst-sci.net/7/617/2007/.

Shvets, A. V., Hayakawa, M., and Maekawa, S.: Results of subionospheric radio LF monitoring prior to the Tokachi $(M=8$, Hokkaido, 25 September 2003) earthquake, Nat. Hazards Earth Syst. Sci., 4, 647-653, 2004, http://www.nat-hazards-earth-syst-sci.net/4/647/2004/.

Sorokin, V. M., Yaschenko, A. K., Chmyrev, V. M., and Hayakawa, M.: DC electric field formation in the mid-latitude ionosphere over typhoon and earthquake regions, Phys. Chem. Earth, 31, 454-461, 2006.

Yoshida, M., Yamauchi, T., Horie, T., and Hayakawa, M.: On the generation mechanism of terminator times in subionospheric VLF/LF propagation and its possible application to seismogenic effects, Nat. Hazards Earth Syst. Sci., 8, 129-134, 2008, http://www.nat-hazards-earth-syst-sci.net/8/129/2008/. 